\title{
Bio-inspired population-based meta-heuristics for problem solving
}

\author{
Jos Manuel Ferrández ${ }^{1} \cdot$ Ramiro Varela $^{2}$
}

Published online: 27 April 2017

(C) Springer Science+Business Media Dordrecht 2017

The International Work-Conference on the Interplay between Natural and Artificial Computation (IWINAC) was started by Professor José Mira in 2005; since then is a biannual conference with focus on the relationship between natural processes and computational models. It is the aim of each IWINAC meeting to bring together scientists and engineers from fields as Electronic Engineering, Artificial Intelligence, Knowledge Engineering, Physics, Mathematics, Computation, Artificial Vision, Situated Robotics, Neurophysiology, Cognitive Science, Linguistics and Philosophy to exchange and put forward new ideas to shed some light on these two questions:

1. What can Physics, Mathematics, Engineering, Computation, Artificial Intelligence and Knowledge Engineering contribute to the understanding of Nervous System, Cognitive Processes and Social Behaviour? This is the scope of Computational Neuroscience and Cognition, which uses computation to model and improve our understanding of natural phenomena.

2. How can Engineering, Mathematics, Computation, Artificial Intelligence and Knowledge Engineering find inspiration in the behaviour and internal functioning of physical, biological and social systems to conceive, develop and build-up new concepts, materials, mechanisms and algorithms of potential value in

Ramiro Varela

ramiro@uniovi.es

Jos Manuel Ferrández

jm.ferrandez@upct.es

1 Department of Electronic and Computers Technology, University of Cartagena, Cartagena, Spain

2 Department of Computer Science, University of Oviedo, Gijón, Spain real world applications? This is the scope of the new Bionics, known as Bioinspired Engineering and Computation, as well as of Natural Computing.

This special issue is more concerned with the second of the above questions and presents applications of bio-inspired computation models as evolutionary algorithms, cuckoo search or bat-inspired optimization to a number of complex real-life problems. It includes five papers which are extended versions of selected papers from the IWINAC 2015 conference held in Elche, in Alicante (Spain) in June 2015. All of them underwent a thorough revision process through two or three rounds of reviews before being definitively accepted. These papers are:

1. "Self-Sampling Strategies for Multimemetic Algorithms in Unstable Computational Environments" by Rafael Nogueras and Carlos Cotta. In this paper, the authors consider island-based multimemetic algorithms running on a network of computing nodes which is not stable as some nodes and links may appear or disappear over time. They propose techniques to endow the system with self-scaling and selfhealing capabilities to redistribute the computations on such unstable environments. To this end, they use probabilistic models to self-sample populations and to rewire the network to keep the connectivity. The proposed model is evaluated on three test problems of numerical optimization.

2. "Evolution of synaptic delay based neural controllers for implementing CPGs in hexapod structures" by José Santos Reyes and Pablo Fernández. The confronted problem is the design of walking controllers in hexapod robots. These controllers are implemented by synaptic delay neural networks which are trained by differential evolution. The authors compare their 
results against continuous recurrent neural networks. Both methods are evaluated on the hexapod robotic structure implemented with the physics simulator Open Dynamics Engine (ODE).

3. "A Binary Cuckoo Search Algorithm for Solving the Set Covering Problem" by Ricardo Soto, Broderick Crawford, Rodrigo Olivares, Jorge Barraza, Franklin Johnson and Fernando Paredes. In this paper the authors tackle the set covering problem, a classical optimization problem with many applications in reallife domains. They combine two bio-inspired metaheuristics as cuckoo search and black-hole optimization. Their approach achieves good results and outperforms some recent proposed methods.

4. "Collaborative Group Formation Using Genetic Algorithms" by Maria Pinninghoff, Miguel Ramirez, Ricardo Contreras Arriagada and Pedro Salcedo Lagos. Here the problem is that of group formation for collaborative learning, a essential problem in many academic activities. The authors propose to use a standard genetic algorithm with permutation encoding, which takes into account the similarities and differences among the individuals to form successful groups. The algorithm is evaluated on three types of classes in the Chilean educational system.
5. "Online Control of Enumeration Strategies via BatInspired Optimization" by Ricardo Soto, Broderick Crawford, Rodrigo Olivares, Franklin Johnson and Fernando Paredes. This paper proposes a strategy for choosing the most promising variable/value ordering heuristic at each step of a search procedure. The solver is first run for a given time using different heuristics, gathering information that is used to build a weightedsum model (WSM). Then the weights of WSM are optimized by means of two meta-heuristics: bat algorithm and black hole optimization. In a second step, the instance is solved choosing at each step the most promising heuristic in accordance with the optimized WSM.

These five papers are good examples of successful application of bio-inspired population-based metaheuristics to solver complex real-life problems. We thank the authors of these papers for their contribution to this special issue and for their patience and collaboration over the review process. Also, we are very grateful to the anonymous reviewers for their work, whose critics and suggestions have helped the authors to improve their papers. Thanks also to editorial staff of Natural Computing for accepting the edition of this special issue and to the IWINAC organizing committees for making this conference possible. 